\title{
Atividade física habitual de crianças e adolescentes mensurada por pedômetro e sua relação com índices nutricionais
}

\author{
Usual physical activity in children and adolescents measured \\ by pedometer and its association with nutritional indicators
}

\author{
Clara Suemi da Costa Rosa ${ }^{1}$ \\ Kelly Patrícia Messias 2 \\ Rômulo Araújo Fernandes 1 \\ Camila Buonani da Silva 2 \\ Henrique Luiz Monteiro ${ }^{3}$ \\ Ismael Forte Freitas Júnior 2
}

1 Universidade Estadual Paulista. Rio Claro, SP. Brasil.

2 Universidade Estadual Paulista. Presidente Prudente, SP. Brasil.

3 Universidade Estadual Paulista. Bauru, SP. Brasil.

Recebido em 27/05/10 Revisado em 17/07/10 Aprovado em 25/10/10
Resumo - A monitoração dos níveis de prática de atividade física em segmentos da população jovem tem se tornado importante tema de interesse entre especialistas da área. O Objetivo do estudo foi verificar o nível de atividade física de escolares, baseando-se em duas recomendações vigentes, e analisar a existência de associações entre a quantidade de passos/dia e diferentes indicadores de adiposidade corporal. A casuística foi composta por 162 indivíduos de dez a 18 anos (65 do sexo masculino e 97 do sexo feminino). Foram calculados o Índice de Massa Corporal e o percentual de gordura por impedância bioelétrica. O nível de atividade física habitual foi mensurado por meio de "pedômetro" (New Lifestyles modelo NL-2000) e analisado de acordo com duas recomendações internacionais: Duncan et al. (masculino: 16.000 passos/dia e feminino: 13.000 passos/dia) e Tudor-Locke et al. (masculino: 15.000 passos/dia e feminino: 12.000 passos/dia). O teste t de Student e a ANOVA One-Away (Post Hoc - LSD) compararam os grupos formados. O nível de significância adotado foi de 5\% (p£0,05). Dos resultados, apenas 18,5\% da amostra cumpriu as recomendações propostas por Duncan e $25,9 \%$ cumpriram os pontos de corte propostos por Tudor-Locke et al.. Os adolescentes que não cumpriram as recomendações propostas por Duncan et al., apresentaram valores percentuais de gordura corporal maiores quando comparados aos jovens que cumpriram $(\mathrm{p}<0,05)$. Pode-se concluir que uma elevada taxa de jovens não cumpriu as duas recomendações analisadas, bem como, apenas um indicador de adiposidade associou-se com o cumprimento de umas das recomendações.

Palavras-chave: Sensor de movimento; Obesidade; Sobrepeso; Adolescente.

Abstract - Monitoring physical activity levels in segments of the young population has become an important topic among specialists. The objective of this study was to analyze the physical activity level of schoolchildren based on two current recommendations and to examine the association between the number of steps/day and different indicators of adiposity. The sample consisted of 162 subjects aged 10 to 18 years (65 boys and 97 girls). Body mass index and body fat percentage were calculated. The level of physical activity was quantified with a pedometer (New Lifestyles NL-2000) based on the following recommendations: Duncan et al. (male: 16,000 steps/day and female: 13,000 steps/day) and Tudor-Locke et al. (male: 15,000 steps/day and female: 12,000 steps/day). The Student $t$-test and one-way ANOVA (LSD post hoc test) were used for comparison between groups. A level of significance of 5\% was adopted ( $p £ 0.05$ ). Only 18.5\% of the sample met the recommendations proposed by Duncan et al. and $25.9 \%$ met the cutoff proposed by Tudor-Locke et al. Adolescents who did not meet the cutoff proposed by Duncan had higher body fat percentages than those who did ( $p<0.05)$. In conclusion, a high rate of the youngsters did not meet the recommendations analyzed and only one indicator of adiposity was associated with meeting one of the recommendations.

Key words: Motion sensor; Obesity; Overweight; Adolescent. 


\section{INTRODUÇÃO}

Sabe-se que o nível de atividade física tem influência sobre a evolução da obesidade, e que a fase da infância e da adolescência são períodos críticos no desenvolvimento de hábitos que, via de regra, serão adotados na vida adulta ${ }^{1,2}$. Dessa forma, experiências positivas com atividade física na infância podem auxiliar o indivíduo a formar base para ser fisicamente ativo quando adulto ${ }^{3,4}$.

A monitoração dos níveis de prática de atividade física em segmentos da população jovem tem se tornado importante tema de interesse entre especialistas da área. Como tentativa de quantificar o nível de atividade física, tem-se utilizado sensores de movimento, como o pedômetro, que apresenta boa aplicabilidade, baixo custo, fácil manuseio, simples utilização e não exige software específico para leitura dos resultados.

Evidências indicam que o acúmulo de 10.000 passos/dia, estimado pelo pedômetro, está relacionado com a manutenção da saúde e controle de peso corporal de pessoas adultas $s^{5,6}$.

No caso de crianças e adolescentes, não há consenso entre os estudos. Tudor-Locke et al. ${ }^{7}$ recomendam o acúmulo de 12.000 passos/dia para o sexo feminino e 15.000 passos/dia para o masculino, pelo menos, cinco dias da semana. Duncan et al. ${ }^{8}$ analisaram a quantidade de passo de crianças sobrepesadas/ obesas e compararam com eutróficas e sugerem 16000 passos/dia para meninos e 13000 passos/dia para meninas, como pontos de corte para classificar os jovens como eutróficos ou excesso de peso.

Apesar dessas recomendações e de estar bem documentado na literatura que baixos níveis de atividade física contribuem com a obesidade na infância ${ }^{9,10}$, ainda são limitados os estudos que descrevem a associação entre a média de passos e a composição corporal em crianças e adolescentes no Brasil.

Assim, o objetivo do presente estudo foi verificar o nível de atividade física de escolares de 10 a 18 anos, baseando-se em duas recomendações para número de passos/dia, bem como, analisar a existência de possíveis associações entre a quantidade de passos/dia e diferentes indicadores de adiposidade corporal.

\section{PROCEDIMENTOS METODOLÓGICOS}

O presente estudo é um delineamento transversal, que foi realizado na cidade de Presidente Prudente, estado de São Paulo. Foram avaliados jovens de ambos os sexos, matriculados de $5^{a}$ série do Ensino
Fundamental aos três anos do Ensino Médio (idade entre 10 a 18 anos) e regularmente matriculados em três escolas públicas estaduais selecionadas por conveniências, uma vez que já possuíam vínculos previamente firmados com a universidade em questão, bem como, disponibilizaram locais apropriados para as avaliações (sala reservada). Como não havia equipamentos para avaliar todos os alunos das escolas envolvidas, em cada uma das escolas, todos os alunos matriculados foram agrupados em um único grupo e, respeitando a proporcionalidade quanto aos grupos etários (10 a 18 anos), sexo e período de estudo (diurno e vespertino), os mesmos foram selecionados de maneira aleatória.

Dos 236 sujeitos sorteados que aceitaram participar do estudo, foram excluídos 74 , que não apresentaram dados referentes a: i) algumas das variáveis necessárias para a análise dos dados (não possuíam ambas: antropometria e análise de impedância bioelétrica [ $\mathrm{n}=15]$ ); e, ii) não permaneceram com o pedômetro durante, pelo menos, três dias $(n=59)$. Dessa forma, a amostra foi constituída por 162 indivíduos de 10 a 18 anos de ambos os sexos, sendo 65 do masculino e 97 do feminino.

Após os devidos esclarecimentos sobre os objetivos e metodologia empregados para a coleta dos dados, e sobre possíveis riscos e benefícios decorrentes da participação na pesquisa, somente aqueles cujos pais ou responsáveis assinaram o "Termo de Consentimento Livre e Esclarecido" foram incluídos no estudo. Essa investigação foi autorizada pelo Comitê de Ética em Pesquisa da FCT/UNESP (processos número 085/2006 e 069/2006).

Foram controladas as variáveis antropométricas: peso corporal, estatura, resistência e reatância por impedância bioelétrica. O peso corporal foi aferido com uma balança digital da marca Filizola, com precisão de $0.1 \mathrm{~kg}$ e capacidade máxima de $150 \mathrm{~kg}$. A estatura, por meio de um estadiômetro, com precisão de $0.1 \mathrm{~cm}$ e extensão de $2 \mathrm{~m}$. Para o cálculo do IMC, dividiu-se o peso corporal (quilogramas) pelo quadrado da estatura (metros), sendo os valores expressos em $\mathrm{kg} / \mathrm{m}^{2}$.

Todas as variáveis antropométricas foram coletadas por avaliadores previamente treinados, e os procedimentos metodológicos adotados são os descritos por Freitas Jr et al. ${ }^{11}$.

A idade cronológica foi estimada levando em consideração a data de nascimento e o dia da avaliação. Para algumas das análises estatísticas, o grupo foi dividido em menor que 15 anos e maior que 14 anos, formando, assim, duas faixas etárias: 10 a 14 anos e 15 a 18 anos. 
Para estimativa da gordura corporal por meio da Impedância Bioelétrica (\%GC-BIA), foi utilizado um aparelho de frequência simples, modelo BIA Analyzer - 101 Q (marca RJL Systems, Detroit, USA) e adotadas as equações propostas por Sun et al. ${ }^{12}$. Apesar de se ter conhecimento de que a equação de Sun et al. foi desenvolvida para pessoas com idade entre 12 a 94 anos, em análises prévias, observamos que esta foi a que apresentou melhor coeficiente de correlação com outros indicadores de gordura corporal, dobra cutânea tricipital e IMC, alem de ser a equação recomendada pelo fabricante do aparelho de impedância bioelétrica, utilizado no presente estudo.

Outras equações que se tem conhecimento e que foram desenvolvidas para crianças e adolescentes $^{13-15}$, também apresentam limitações com relação à etnia e à própria faixa de idade. Além disso, o principal fator determinante para a recusa destas outras equações foi a variação de valores que, notadamente, subestimam o percentual de gordura para aqueles que apresentam valores muito baixos.

Os escolares foram avaliados na própria escola, em decúbito dorsal, durante as primeiras horas da manhã, após urinar e sem utilizar nenhum material metálico (brinco, pulseira, etc.). Os jovens foram aconselhados a não realizarem atividades físicas vigorosas no dia anterior à avaliação, e todos os procedimentos adotados para a realização do teste são descritos por Heyward e Storlakzik ${ }^{16}$. Na amostra total, 40 sujeitos não possuíam os valores referentes à análise de impedância bioelétrica (erros de leitura do aparelho, falta no dia da avaliação ou não cumprir todas as recomendações para a realização do teste ${ }^{16}$ ), porém, possuíam os valores de antropometria e, dessa forma, foram mantidos no estudo e as análises para impedância bioelétrica foram realizadas com 122 sujeitos.

O nível de atividade física habitual foi avaliado por meio de um sensor de movimento, conhecido como "pedômetro", que registra as oscilações verticais do corpo. Dessa forma o valor registrado é referente ao número de passos realizados pelo avaliado. Os pedômetros utilizados no estudo (marca New Lifestyles modelo NL-2000) possuem memória interna que registra os dias da semana analisados, bem como o montante de passos/dia. Assim, sempre na segunda-feira, os mesmos foram instalados junto à cintura do avaliado e selados com lacre de plástico que foi violado apenas no dia da coleta dos dados (na segunda-feira seguinte), após sete dias de utilização, com remoção do equipamento apenas para tomar banho e dormir.
Caso o avaliado praticasse algum tipo de esporte aquático, foi instruído a retirar o pedômetro enquanto estivesse dentro do meio liquido. Este aparelho tem capacidade de armazenamento de dados de sete dias e de todos os dados registrados, foram considerados somente aqueles realizados nos dias completos, motivo pelo qual foram descartados os registros do primeiro dia. Estudo publicado por Tudor-Locke et al. ${ }^{17}$ indica que a mensuração de apenas três dias condiz com a média diária de passos de uma pessoa $(r=0,80)$. Então, foram incluídos na amostra apenas os indivíduos que utilizaram pelo menos 2 dias da semana e 1 dia do final de semana. Números de passos abaixo de 1000 ou acima de 30.000 foram descartados da amostra por serem considerados falsos ${ }^{18}$.

Assim, em nosso estudo, dois pontos de corte foram adotados, aqueles propostos por Tudor-Locke et al. (Feminino: 12.000 passos/dia e Masculino: 15.000 passos/dia) e aqueles propostos por Duncan et al. ${ }^{8}$ (Feminino: 13.000 passos/dia e Masculino: $16.000 \mathrm{passos} / \mathrm{dia})$.

$\mathrm{O}$ teste de Komolgorov-Smirnov indicou que todos os conjuntos de dados analisados se enquadraram no modelo de distribuição normal. Foram calculados valores de média e desvio-padrão como estatística descritiva. O teste "t de Student" para dados independentes estabeleceu comparações entre os adolescentes que cumpriram e não cumpriram as duas recomendações para a prática de atividades físicas. Para análise das possíveis diferenças entre as médias, envolvendo mais de três grupos, foi utilizada a ANOVA One-Away (Post Hoc LSD). O nível de significância adotado para todos os procedimentos estatísticos foi de 5\% (p£0,05).

\section{RESULTADOS}

Na tabela 1, estão dispostas as características gerais da amostra: percentual de alunos por série em cada escola, percentual de indivíduos estratificada por idade e sexo e média e desvio-padrão do IMC e $\%$ GC-BIA.

As características gerais da amostra estratificadas de acordo com sexo e mediana da idade encontram-se descritas na tabela 2 .

Observa-se que na média de passos por dia, o grupo meninos $\leq 14$ anos apresentou melhor desempenho quando comparado ao grupo masculino $\geq 15$ anos, bem como aos dois grupos do sexo feminino.

A média de passos/dia para o sexo masculino (11.851 \pm 4.544$)$, independente da faixa etária, foi estatisticamente significante $(p \leq 0,05)$ em rela- 
ção ao feminino, que apresentou uma média de $9.424 \pm 3.620$ passos/dia, ou seja, 20,4\% menos passos que os meninos.

A Figura 1 apresenta o percentual de jovens que cumpriram, ou não, as recomendações para a prática habitual de atividades físicas. Para o sexo masculino, apenas 20\% atendeu à média de 16000 passos/dia propostos por Duncan et al. ${ }^{8}$ e $29,2 \%$ atingiram a média 15000 passos/dia proposto por Tudor-Locke et al. ${ }^{7}$. O percentual para o sexo feminino foi mais baixo, apenas 17,5\% cumpriram média de passos/dia de 13000 proposto por Duncan et al. ${ }^{8}$ e 23,7\% cumpriram média de passos/dia de 12000 proposto por Tudor-Locke et al.?
Na Tabela 3, são apresentadas as comparações entre indivíduos que cumpriram e não cumpriram as recomendações propostas no que se refere aos indicadores de adiposidade utilizados.

Os adolescentes que não cumpriram as recomendações vigentes apresentaram valores maiores de adiposidade corporal quando comparados aos que cumpriram, porém, apenas o \%GC-BIA nos pontos de corte de Duncan et al. ${ }^{8}$, apresentou significância estatística nas comparações.

\section{DISCUSSÃO}

Trata-se de estudo transversal que buscou analisar

Tabela 1. Características gerais da amostra: média e desvio-padrão do IMC e \%GC-BIA, e percentual de alunos por idade, sexo e série em cada escola.

\begin{tabular}{|c|c|c|c|c|}
\hline & \multicolumn{2}{|c|}{ Masculino } & \multicolumn{2}{|c|}{ Feminino } \\
\hline & $\leq 14$ anos & $\geq 15$ anos & $\leq 14$ anos & $\geq 15$ anos \\
\hline N (\%) & 63,1 & 36,9 & 43,3 & 56,7 \\
\hline \multirow[t]{2}{*}{$\% G C-B I A^{\S}$} & $23,5 \pm 7,8$ & $21 \pm 5$ & $28,1 \pm 7,8$ & $33,2 \pm 5,2$ \\
\hline & Escola A & \multicolumn{2}{|c|}{ Escola B } & Escola C \\
\hline N Ensino Médio (\%) & 44,7 & \multicolumn{2}{|c|}{100} & 30,3 \\
\hline
\end{tabular}

Nota. $\leq 14$ anos: grupo etário com idade $\leq 14$ anos; $\geq 15$ anos: grupo etário com idade $\geq 15$ anos; N: número de indivíduos, IMC índice de massa corporal; \%GC-BIA: percentual de gordura calculado por meio da impedância bioelétrica. ; § $\mathrm{n}=122$ participantes; N Ensino Fundamental: número de indivíduos na série escolar ginasial; N Ensino Médio.

Tabela 2. Valores de média, desvio-padrão de passos/dia, do sexo feminino e masculino para cada faixa etária, nível de significância e diferenças entre os grupos.

\begin{tabular}{lcc}
\hline \multirow{2}{*}{ Faixa etária } & \multicolumn{2}{c}{ Sexo } \\
\cline { 2 - 3 } & Masculino $(\mathrm{n}=65)$ & Feminino $(\mathrm{n}=97)$ \\
\hline \multirow{2}{*}{$\leq 14$ anos } & $12514 \pm 4607^{\mathrm{Bb}}$ & $9502 \pm 3477^{\text {Aa }}$ \\
& $(\mathrm{n}=41)$ & $(\mathrm{n}=42)$ \\
& $10716 \pm 4291^{\text {Aa }}$ & $9364 \pm 3755^{\text {Aa }}$ \\
& $(\mathrm{n}=24)$ & $(\mathrm{n}=55)$ \\
\hline
\end{tabular}

Nota. $\leq 14$ anos: grupo etário com idade $\leq 14$ anos; $\geq 15$ anos: grupo etário com idade $\geq 15$ anos; $\mathrm{n}=$ número de indivíduos. Letras maiúsculas - fixada a variável sexo compararam as faixas etárias (B>A); Letras minúsculas - fixada a variável faixa etária compararam sexo $(b>a)$.
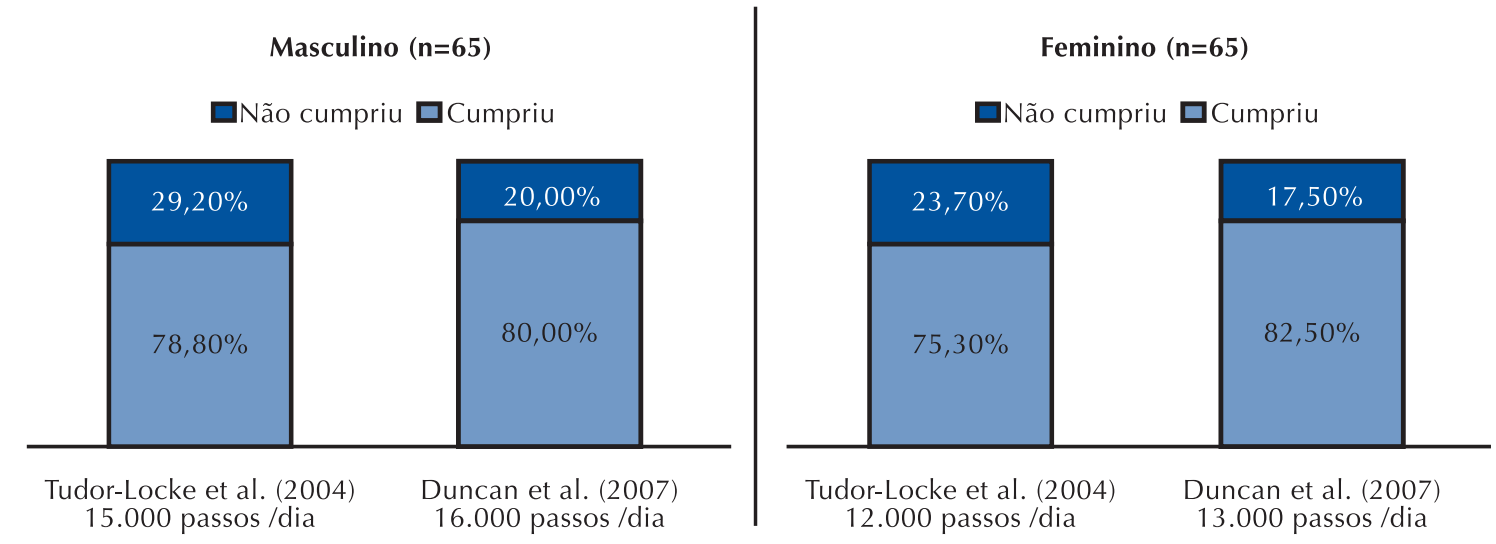

Figura 1. Distribuição percentual das crianças e adolescentes por sexo, que cumpriram e não cumpriram o número de passos/ dia, segundo valores de corte propostos por Tudor-Locke et al. ${ }^{7}$ e Duncan et al. ${ }^{8}$. 
Tabela 3. Indicadores de adiposidade estratificados de acordo com a prática de atividades físicas entre adolescentes.

\begin{tabular}{|c|c|c|c|c|c|}
\hline \multirow{2}{*}{ Ponto de Corte } & \multirow{2}{*}{ Variáveis } & \multicolumn{2}{|c|}{ Frequência média de passos/dia } & \multirow{2}{*}{ t de student } & \multirow{2}{*}{$P$ value } \\
\hline & & Cumpriu & Não cumpriu & & \\
\hline \multirow{3}{*}{$\begin{array}{l}{ }^{1} 16000^{1} \\
\$ 13000\end{array}$} & $\mathrm{~N}$ & 30 & 132 & & \\
\hline & $\mathrm{IMC}\left(\mathrm{kg} / \mathrm{m}^{2}\right)$ & $19,68 \pm 3,1$ & $20,63 \pm 3,6$ & $-1,340$ & 0,182 \\
\hline & $\% G C-B I A^{\S}$ & $24,75 \pm 7,7$ & $28,42 \pm 7,9$ & $-1,995$ & $0,048^{*}$ \\
\hline \multirow{3}{*}{$\begin{array}{l}\mathrm{O}^{1} 15000^{2} \\
\text { \$1 } 12000\end{array}$} & N & 42 & 120 & & \\
\hline & $\operatorname{IMC}\left(\mathrm{kg} / \mathrm{m}^{2}\right)$ & $19,83 \pm 3,2$ & $20,67 \pm 3,5$ & 0,391 & 0,165 \\
\hline & $\% G C-B I A^{\S}$ & $25,98 \pm 7,3$ & $28,32 \pm 8,2$ & $-1,470$ & 0,162 \\
\hline
\end{tabular}

Nota. 1: Duncan et al. (2007); ${ }^{2}$ : Tudor-Locke et al. (2004); IMC: índice de massa corporal; \%GC-BIA: percentual de gordura calculado por meio da impedância bioelétrica. ; ${ }^{\varsigma}: \mathrm{n}=122$ participantes; * $\mathrm{p}<0,05$.

a prática habitual de atividade física, estimada pela pedometria, em crianças e adolescentes de ambos os sexos, assim como o relacionamento com adiposidade corporal.

Na presente pesquisa, a quantidade de passos/ dia no sexo masculino (11.850) e feminino (9.424) foi inferior àquela observada em populações pediátricas de outros países. Vicent et al. ${ }^{19}$,analisando crianças de 6 a 12 anos da Suécia, Austrália e Estados Unidos da América, observaram que nas suecas, a média de passos variou de 15.673 a 18.346 no caso do sexo masculino e de 12.041 a 14.825 passos para o feminino. Nas australianas, a média de passos variou de 13.864 a 15.023 para os meninos e de 11.221 a 12.322 para as meninas, já as crianças americanas apresentaram menor média (masculino: 12.554 a 13.872 e feminino: 10.661 a 11.383 ), mas ainda assim, maiores do que a média observada em nossa investigação. Não há estudos nacionais envolvendo pedometria, no entanto, pesquisadores brasileiros, utilizando-se de outros instrumentos, têm observado elevada prevalência de inatividade física entre adolescentes brasileiros ${ }^{20,21}$. Adicionalmente, em estudo anterior envolvendo adolescentes da cidade de Presidente Prudente, também se constatou elevada prevalência de inatividade física ${ }^{22}$.

A baixa quantidade de passos diários observada na amostra do presente estudo se confirma ao classificar estes jovens de acordo com as duas recomendações para a prática de atividades físicas encontradas na literatura. No grupo masculino, 70,8\% não cumpriram recomendação proposta por Tudor-Locke et al. ${ }^{7}$ e $80 \%$ por Duncan et al. ${ }^{8}$. No caso do sexo feminino, esses percentuais foram maiores $76,3 \%$ e $82,5 \%$, respectivamente, de acordo com os dois autores citados. Estas recomendações são utilizadas em outros países, fato este que possibilita comparações. Al-Hazzaa ${ }^{23}$,em 2007, verificou que 61,3\% de uma amostra de 296 meninos (8-12 anos de idade) não atingiu os valores de corte propostos por Tudor-Locke et. al? (15.000 passos/dia); este resultado é similar ao percentual de meninos que não cumpriram os pontos de corte no presente estudo.

A média de passos/dia observada para o sexo masculino foi estatisticamente superior em relação ao sexo feminino. Estes dados estão de acordo com outros estudos encontrados na literatura técni$\mathrm{ca}^{18,24-26}$. Uma possível explicação para tal fato foi relatada em estudo de Gonçalves et al..$^{27}$ quando observaram que meninos têm suporte/incentivo familiar maior para a prática de atividades físicas e, por este motivo, participam mais de atividades físicas vigorosas, comparativamente às meninas.

A média de passos observada nos diferentes grupos etários investigados no presente estudo apresenta similaridade em relação ao descrito na literatura ${ }^{26,28,29}$, onde meninos de menor faixa etária apresentaram maior número de passos quando comparados aos de maior idade. $\mathrm{O}$ trabalho publicado pelo President's Council on Physical Fitness and Sports ${ }^{28}$ reuniu dados de Vincent e Pangrazi (2002) (amostra de pré-escolares), de Lê Masurier (2004) (crianças de 7 a 8 anos) e Wilde et. al. (2004) (crianças de 10 a 12 anos), os quais indicaram o declínio no número de passos/dia com o avanço da idade. Pate et al. ${ }^{30}$ também observaram que adolescentes do sexo feminino apresentam declínio anual de $4 \%$ na prática de atividades físicas quando comparadas às crianças de menor idade. Os motivos relacionados a este declínio ainda não são claros e é motivo de preocupação, uma vez que a prática deste tipo de atividade na infância e adolescência tende a se perpetuar até a vida adulta ${ }^{2}$.

Os dados apresentados indicaram que, cumprir ambas as recomendações para a prática de atividades físicas baseadas em pedometria se associou pouco com os indicadores de adiposidade, onde, apenas a recomendação de Duncan et al. ${ }^{8}$ associou-se com a impedância bioelétrica. Outros autores encontraram associações mais fortes que a nossa. Moraes et al..$^{31}$ identificaram que adolescentes que 
não cumprem as recomendações para a prática de atividades físicas apresentam maior taxa de obesidade. A esse respeito, estudo realizado por Fernandes et al. ${ }^{32}$, na cidade de Presidente Prudente, observou elevadas taxas de sobrepeso/obesidade, que, por sua vez, coexistem com uma elevada prevalência de adolescentes com baixa prática de atividades físicas ${ }^{32}$.

O presente estudo apresenta pontos positivos, pois, no que se refere ao uso de pedômetros, não há similaridade na literatura nacional e, além disso, os equipamentos utilizados para avaliar o nível de atividade física praticada permitiram fazer uma estimativa da quantidade média semanal de passos, diminuindo, assim, a possibilidade de erros de medida por parte do avaliador; esta segunda característica confere ao estudo uma significativa qualidade metodológica. Por outro lado, suas principais limitações precisam ser destacadas. $\mathrm{O}$ desenho de estudo, de caráter transversal, impossibilita verificar se o excesso de gordura corporal de crianças e adolescentes é decorrente da atividade física habitual. A alta taxa de exclusões (próxima de 30\%) gerada pela utilização do pedômetro deve ser considerada na análise dos resultados, pois não foi possível comparar este grupo excluído com os mantidos no estudo. Além disso, mesmo se utilizando amostragem aleatória, a não estimação de um cálculo amostral prévio limita o potencial de inferência dos dados.

\section{CONCLUSÃO}

Baseado em ambas as recomendações utilizadas, a amostra deste estudo, independentemente do sexo, apresentou alta proporção de jovens que não cumprem os pontos de corte para passos/dia, o que pode ser um fator determinante para o desenvolvimento de agravos à saúde. Além disso, a pouca associação observada entre passos/dia e adiposidade corporal indica a necessidade da realização de novos estudos nacionais abordando o uso de pedômetros em populações pediátricas.

\section{REFERÊNCIAS BIBLIOGRÁFICAS}

1. Dwyer T, Magnussen CG, Schmidt MD, Ukoumunne OC, Ponsonby AL, Raitakari OT, et al. Decline in physical fitness from childhood to adulthood associated with increased obesity and insulin resistance in adults. Diabetes Care 2009;32:683-7.

2. Azevedo MR, Araújo CL, Cozzensa da Silva M, Hallal PC. Tracking of physical activity from adolescence to adulthood: a population-based study. Rev Saúde Pública 2007;41(1):69-75.

3. Hallal PC, Wells JC, Reichert FF, Anselmi L, Victora CG. Early determinants of physical activity in adolescence: prospective birth cohort study. BMJ Clinical research 2006;332:1002-7.
4. Azevedo MR, Araújo CL, Silva MC, Hallal PC. Tracking of physical activity from adolescence to adulthood: a population-based study. Rev Saúde Pública 2007;41(1):69-75.

5. Tudor-Locke C, Williams JE, Reis JP, Pluto D. Utility of pedometers for assessing physical activity: convergent validity. Sports Med 2002;32(12):795-808.

6. Tudor-Locke C, Bassett Jr DR. How many steps/day are enough? Preliminary pedometer indices for public health. Sports Med 2004;34(1):1-8.

7. Tudor-Locke C, Pangrazi RP, Corbin CB, Rutherford WJ, Vincent SD, Raustorp A, et al. BMI-referenced standards for recommended pedometer-determined steps/day in children. Prev med 2004;38(6):857-64.

8. Duncan JS, Schofield G, Duncan EK. Step count recommendations for children based on body fat. Prev med 200744(1):42-4

9. Pino RA, Petroski EL. Adiposidade corporal e nível de atividade física em adolescenetes. Rev Bras Cineantropom Desempenho Hum 1999;1(1):60-8.

10. Fernandes RA, Nogueira A, Rosa CSC, Bonfim MR, Freitas Jr IF. Hábito alimentar e nível de prática de atividade física de meninos eutróficos e de obesos. Rev Educ Fís/UEM. 2006;17(1):45-51.

11. Freitas Jr IF. Padronização de Técnicas Antropométricas. $1^{a}$ ed. São Paulo: Cultura Acadêmica;2009.

12. Sun SS, Chumlea WC, Heymsfield SB, Lukaski HC, Schoeller D, Friedl K, et al. Development of bioelectrical impedance analysis prediction equations for body composition with the use of a multicomponent model for use in epidemiologic surveys. Am J clin Nutr 2003;77(2):331-40.

13. Houtkooper, L. B., Going, S. B., Lohman, T. G., Roche, A. F., \& Van Loan, M. (1992). Bioelectrical impedance estimation of fat-free body mass in children and yuoth: a cross-validation study. J Appl Phys 72(1):366-73.

14. Lohman, T. G. Advances in Body Composition Assessment. Champaign, IL: Human Kinetics; 1992.

15. R. F. Kushner. Bioelectrical impedance analysis: a review of principles and applications. J Am Coll Nutr 1992;1(2):199-209.

16. Heyward VH, Stolarczyk LM. Applied body composition assessment. Champaign, Illinois: Human Kinetics Books; 1996.

17. Tudor-Locke C, Burkett L, Reis JP, Ainsworth BE, Macera CA, Wilson DK. How many days of pedometer monitoring predict weekly physical activity in adults? Prev Med 2005;40(3):293-8

18. Duncan JS, Schofield G, Duncan EK. Pedometer-determined physical activity and body composition in New Zealand children. Med Sci Sports Exerc 2006;38(8): 1402-9.

19. Vincent SD, Pangrazi RP, Raustorp A, Tomson LM, Cuddihy TF. Activity levels and body mass index of children in the United States, Sweden, and Australia. Med Sci Sports Exerc 2003;35(8):1367-73.

20. Hallal PC, Bertoldi AD, Goncalves H, Victora CG. [Prevalence of sedentary lifestyle and associated factors in adolescents 10 to 12 years of age]. Cadernos de saude publica / Ministerio da Saude, Fundacao Oswaldo Cruz, Escola Nacional de Saude Publica. 2006;22(6):1277-87. 
21. Ceschini FL, Andrade DR, Oliveira LC, Araújo Júnior JF, Matsudo VK. Prevalence of physical inactivity and associated factors among high school students from state's public schools. J Pediatr 2009;85(4):301-6

22. Fernandes RA, Junior IF, Cardoso JR, Vaz Ronque ER, Loch MR, de Oliveira AR. Association between regular participation in sports and leisure time behaviors in Brazilian adolescents: a cross-sectional study. BMC Public Health 2008;8(329).

23. Al-Hazzaa AM. Pedometer-determined Physical Activity among obese and non-obese 8 - to 12 - year-old Saudi Schoolboys. J Physiol Anthropol 2007;26(4):459-65.

24. Rowlands AV, Eston RG, Ingledew DK. Relationship between activity levels, aerobic fitness, and body fat in 8 to 10-yr-old children. J Appl Physiol 1999;86(4):1428-35.

25. Vincent SD, Pangrazi RP. An examination of the activity patterns of elementary school children. Pediatr Exerc Sci 2002;14:432-41.

26. Raustorp A, Svenson K, Perlinger T. Tracking of pedometer-determined physical activity: a 5-year follow-up study of adolescents in Sweden. Pediatr Exerc Sci 2007;19(2):228-38.

27. Gonçalves H, Hallal PC, Amorim TC, Araújo CL, Menezes AM. Sociocultural factors and level of physical activity in early adolescence. Rev Panam Salud Publica. 2007;22:246-53.

28. Vincent SD, Sidman CL. Determining Measurement Error in Digital Pedometers. Meas Phys Educ Exerc Sci 2003;7(1):19-24.
29. President's Council on Physical Fitness and Sports. Physical Activity for Children: Current Patterns and Guidelines. 2004;5(2).

30. Pate RR, Stevens J, Webber LS, Dowda M, Murray DM, Young DR, et al. Age-related change in physical activity in adolescent girls. J Adolesc Health 2009;44 (3):275-82.

31. Moraes AC, Fernandes CA, Elias RG, Nakashima AT, Reichert FF, Falcão MC. Prevalência de inatividade física e fatores associados em adolescentes. Rev Assoc Med Bras 2009;55(5):523-8.

32. Fernandes RA, Casonatto J, Christofaro DGD, Ronque ERV, Oliveira AR, Freitas Júnior IF. Riscos para o excesso de peso entre adolescentes de diferentes classes socioeconômicas. Rev Assoc Med Bras 2008;54:334-8.

\section{Endereço para correspondência}

Endereço para correspondência

Clara Suemi da Costa Rosa

Caixa Postal 819

CEP: 19814-970 - Assis, SP. Brasil

E-mail: clarasuemi@hotmail.com 